\title{
CULPAS NO MÉDICAS DE LOS HOSPITALES PÚBLICOS
}

\author{
José Miguel Valdivia ${ }^{1}$
}

Resumen: La culpa es un elemento central de la responsabilidad de los hospitales públicos en Derecho público chileno. Tratándose de daños provocados por comportamientos no médicos, la culpa se presenta normalmente de modo trivial. El artículo analiza las categorías típicas de esta responsabilidad, que corresponden a fallas en los cuidados al paciente (incluyendo defectos de vigilancia), desórdenes administrativos y deficiencia o insuficiencia de los equipos. Los jueces suelen abordar estos casos como si se tratara de faltas administrativas corrientes.

Palabras clave: malpraxis, hospitales públicos, responsabilidad legal

\section{Non-medical malpractice in public hospitals}

Abstract: Negligence is a key element of public hospitals' responsibility under Chilean public law. When damages are provoked by non-medical misconducts, negligence is often shown under an unspecific face. This paper analyses the typical categories of this kind of liability, namely improper care (including failure to monitor patients), administrative dysfunctions, insufficient or defective devices. Judges normally look these cases as if they were ordinary administrative faults.

Key words: malpractice, hospitals, public, liability legal

\section{Culpas não médicas dos hospitais públicos}

Resumo: A culpa é um elemento da responsabilidade dos hospitais públicos no Direito público chileno. Tratando-se de danos provocados por comportamentos náo médicos, a culpar se apresenta normalmente de modo trivial. $\mathrm{O}$ artigo analisa as categorias típicas desta responsabilidade, que correspondem a falhas nos cuidados ao paciente (incluindo defeitos de vigilância), desordens admnistrativas e deficiência ou insuficiência das equipes. Os juízes geralmente abordam estes casos como se fossem faltas administrativas comuns.

Palavras chave: má prática, hospitais públicos, responsabilidade legal

\footnotetext{
${ }^{1}$ Doctor en derecho (U. París II). Profesor de Derecho administrativo, Facultad de Derecho, Universidad de Chile, Santiago, Chile Correspondencia: jmvaldivia@derecho.uchile.cl
} 


\section{Introducción}

La preocupación ética de no dañar — revitaliza$\mathrm{da}$, en el plano de las atenciones de salud, por el principio ético de nonmaleficence (1:189) — tiene su correlato jurídico en la institución de la responsabilidad civil, esto es, en la indemnización de los perjuicios causados. Más allá de aquella que recae sobre cada profesional o agente en el campo de la salud, también los establecimientos hospitalarios deben hacer frente a estas responsabilidades. En Chile, en el régimen de responsabilidad aplicable a los establecimientos públicos de salud, el concepto central es el de falta de servicio, que corresponde a una especie de culpa institucional (Ley 19.966, artículo 38). La definición de esa culpa exige atender a los deberes del hospital en todas las circunstancias, sea que se refieran a la intervención del médico o a la de otros profesionales o agentes. Es útil deslindar estos dos campos porque la noción de "culpa médica" - dominada por su vinculación con la idea de lex artis - presenta una especificidad cierta(2). La culpa no médica, en cambio, presenta en principio un carácter menos distintivo frente a otras culpas de organismos administrativos.

Por de pronto, como ha notado la jurisprudencia, no corresponde hacer jugar la noción de lex artis en el juzgamiento de las responsabilidades derivadas del desempeño del personal no médi$\mathrm{Co}^{2}$. Sin embargo, el deber de respeto a la integridad física de los pacientes, su dignidad o autonomía, que tiene hondas raíces éticas, se prolonga hacia la totalidad de la institución hospitalaria, incluidos los agentes no médicos. Los deberes de servicio exigibles del hospital público en relación con las actividades de ese personal o de la gestión administrativa tienen una mayor simplicidad, que facilita su revisión por jueces no expertos en medicina.

Ahora bien, las situaciones típicas que se presentan al interior de hospitales y centros de salud por cierto que imprimen notas singulares a la responsabilidad. Este artículo, que prolonga el análisis de la responsabilidad hospitalaria iniciado en un estudio previo(3), se propone revisar esas hipótesis típicas. Para efectos analíticos, conviene separar aquí aquellos casos que se refieren más

${ }^{2}$ Corte Suprema, 6 de noviembre de 2012, Rol 5.417-2012. bien al desempeño del personal de aquellos que conciernen a la inadecuación del equipamiento físico del hospital.

\section{Las culpas del personal no médico}

En los establecimientos hospitalarios laboran, aparte del estamento médico, numerosas otras personas, con calificaciones más o menos exigentes relativas al tratamiento de salud brindado a pacientes, o relativas a simples tareas administrativas. Las faltas de servicio pueden situarse en todo nivel. Solo para efectos esquemáticos, conviene separar los dos ámbitos más significativos en que se agrupan estas culpas, que corresponden al de los cuidados brindados al paciente, con especial detención en los defectos de vigilancia, y las gestiones más bien administrativas del hospital.

\section{La culpa en los cuidados del paciente}

En aquellas tareas relativas a la atención de pacientes que solo pueden confiarse a determinados profesionales, la culpa está sujeta a calificaciones relativamente similares a la culpa médica $(4: 104)^{3}$; por cierto, la entidad de los deberes anejos a estas profesiones no guarda comparación con la complejidad de los deberes médicos. Un ejemplo lo provee el caso Fuentes Figueroa, en que una matrona no valora adecuadamente el cuadro clínico de un neonato ${ }^{4}$.

Con todo, en general, la culpa en este campo viene determinada por estándares prudenciales, cuyo establecimiento no presenta gran dificultad. No es muy concebible, por ejemplo, que con ocasión de las curaciones practicadas sobre el cuerpo de una paciente, una enfermera aplique agua hirviendo en su zona genital provocándole heridas ${ }^{5}$. Tampoco, que el traslado de un paciente en camilla se efectúe en condiciones que permitan su caída(3). En varios casos, el juicio de responsabilidad depende de la calificación de

\footnotetext{
${ }^{3}$ Más allá de la medicina, el recurso a la idea de la lex artis o reglas o usos normativos del oficio es un rasgo común a los regímenes de responsabilidad por culpa en el ejercicio de actividades profesionales (de abogados, contadores, arquitectos, etc.).

${ }^{4}$ Corte Suprema, 9 de enero de 2017, Rol 31.492-2016.

${ }^{5}$ Corte Suprema, 4 de septiembre de 2012, Rol 8.044-2010; la culpa se califica, discutiblemente, como falta personal, aunque el Estado (Hospital Naval de Talcahuano) responde igualmente.
} 
conductas más o menos sencillas a ojos de un profano, relativamente similares a las que pueden observarse en ámbitos de acción distintos de la salud pública.

\section{En especial, los defectos de vigilancia}

Un número significativo de faltas de servicio se aprecia en la vigilancia de los pacientes y su estado de salud. Estas faltas de servicio pueden cometerse desde el ingreso del paciente al recinto hospitalario y aun antes de recibir atención ${ }^{6}$. De modo más trivial, la insuficiente vigilancia de la evolución del paciente - por ejemplo, un neonato ${ }^{7}$ o una parturienta ${ }^{8}$ - configura una falta de servicio.

Un caso que conviene poner aparte, precisamente por su relativa complejidad, es el de los cuidados dispensados a pacientes psiquiátricos. Aquí los deberes del hospital comprenden usualmente la custodia y vigilancia de estos pacientes, que a menudo son desafiadas por su voluntad de escapar o, incluso, sus tendencias suicidas. Los defectos de vigilancia deben establecerse a la luz del cuadro del paciente y de su historial (esto es, de la previsibilidad del daño), así como de los medios del hospital. La personalidad o patología del paciente psiquiátrico imprime una nota de originalidad a los deberes de servicio, que no se presenta en otros casos. Por eso mismo, el estándar de vigilancia exigible no puede ser idéntico en hospitales que cuenten con servicios especializados en el tratamiento psiquiátrico del que se espera de hospitales generalistas?

\section{Faltas en la gestión administrativa del hospital}

Por último, las faltas de servicio más fácilmente reconocibles en el hospital tienen por rasgo en común un desorden en la gestión administrativa del establecimiento, más o menos vinculado con la atención de pacientes.

Así, la irregular distribución del trabajo al interior del hospital, con el resultado de que personal

${ }^{6}$ Corte Suprema, 25 de octubre de 2016, Rol 22.751-2015 (infarto sufrido en los alrededores de la sala de espera).

${ }^{7}$ Corte Suprema, 7 de noviembre de 2016, Rol 52.870-2016.

${ }^{8}$ Corte Suprema, 3 de enero de 2017, Rol 47.886-2016.

${ }^{9}$ Corte Suprema, 25 de julio de 2016, Rol 1.511-2016 (suicidio de paciente herido, atendido de urgencia en hospital general). no calificado aborde tareas propias de los médicos, revela una inequívoca falta de servicio ${ }^{10}$.

En el plano de la gestión, también configuran faltas de servicio los desórdenes que conducen a que un paciente que espera ser atendido simplemente no lo sea ${ }^{11}$.

En fin, defectos de vigilancia similares a los que se aprecian en la atención de pacientes se detectan también en la custodia de los resultados de una intervención o procedimiento hospitalario. Esta labor puede estimarse, en términos muy generales, como una tarea puramente administrativa, de suerte que el extravío de un feto ${ }^{12}$, de una biopsia $^{13}$, de la ficha clínica del paciente ${ }^{14}$ o de los resultados de un examen - así como, en general, los desórdenes en la entrega de exámenes ${ }^{15}$ — son hipótesis ordinarias de falta de servicio.

\section{La inadecuación de los medios}

A veces, en el origen de un accidente hospitalario está una situación puramente objetiva, vinculada a los instrumentos, de cualquier naturaleza, con que el hospital cumple sus funciones, e incluso el estado físico de sus instalaciones. Merecen analizarse tres series de situaciones distintas.

\section{Defectos del instrumental}

En Rojas Contreras la falta de servicio es reconocida por la utilización de instrumentos dentales de los que sale un líquido corrosivo en vez de agua $^{16}$. Cabe pensar que la solución es extensible a otros casos en que el accidente se deba al mal estado de las cosas empleadas en la atención del paciente, desde los utensilios y la maquinaria de

\footnotetext{
${ }^{10}$ Recientemente, Corte Suprema, 29 de junio de 2018, Rol 35.7212017 (interpretación de exámenes y formulación de diagnóstico por una matrona).

${ }^{11}$ Corte Suprema, 2 de marzo de 2017, Rol 34.842-2016 (orden de derivación de un hospital a otro incumplida, sin que el hospital derivado hubiera puesto en lista de espera al paciente).

${ }^{12}$ Corte Suprema, 29 de abril de 2004, Rol 4.212-2002.

${ }^{13}$ Corte Suprema, 13 de enero de 2011, Rol 363-2009.

${ }^{14}$ Corte Suprema, 28 de abril de 2014, Rol 2.509-2014.

${ }^{15}$ Por ejemplo, Corte Suprema, 22 de septiembre de 2016, Rol 9.481-2016 (caso del Hospital de Iquique, que omitió notificar los resultados de exámenes de VIH a unas 25 personas, entre otros la víctima).

${ }^{16}$ Corte Suprema, 19 de octubre de 2016, Rol 49.707-2016.
} 
uso médico ${ }^{17}$ hasta los productos que deben emplearse en la atención o cuidado de los pacientes (medicamentos, desinfectantes, productos sanguíneos, etc.).

La simplicidad del mecanismo de responsabilidad parece obedecer a una presunción de falta de servicio construida a partir del estado defectuoso del objeto. Ese mal estado revela que en la adquisición, elaboración o mantención de esos objetos o equipos se ha cometido una falta de servicio. Por cierto, para que la presunción opere se requiere que la culpa presunta haya podido estar dentro del ámbito de deberes del hospital (lo que no ocurriría respecto de productos médicos elaborados fuera del hospital y que no pueden ser inspeccionados antes de su uso).

\section{Déficit del instrumental}

Los defectos del instrumental o insumos guardan semejanza con su insuficiencia cuantitativa (contar con instrumento defectuoso puede equivaler a no tenerlo). Con todo, a menudo juegan un papel causal distinto: mientras el mal estado de una cosa suele ser causa directa del daño, la ausencia de materiales solo impide su evitación. Por ejemplo, en Ponce Carrasco la falta de un tomógrafo que hubiera permitido la práctica de un scanner, por estar "descompuesto desde hacía ya varios días", se juzga como constitutiva de falta de servicio ${ }^{18}$.

En estos casos - como en otros relativos a omisiones - lo jurídicamente relevante consiste en saber si algún estándar de servicio exige al hospital contar con tal objeto. En principio, el desajuste entre el equipamiento exigible y el realmente disponible puede revelar una mala organización, que es un caso típico de falta de servicio.

Ahora bien, la responsabilidad no debe derivar en una estigmatización sistemática de los hospitales pobres (que, para aquellos de dimensiones

\footnotetext{
${ }^{17}$ En un antiguo caso, Cancino Rojas, la responsabilidad es acogida, bajo el pretexto de negligencia del equipo médico, aparentemente en razón de la inoculación al paciente residuos provenientes del lavado de jeringas: Juzgado de Talca, 26 de mayo de 1989, en: Revista Ius et Praxis 1996; 2(1): 125 y s.; confirmada por Corte de Apelaciones de Talca, 25 de septiembre de 1990 y Corte Suprema, 9 de mayo de 1991, en: Gaceta Jurídica 1991; 131: 78.

${ }^{18}$ Corte Suprema, 15 de mayo de 2012, Rol 9.145-2009.
}

o complejidad más reducidas importaría una especie de culpa por existir). Si la falta de servicio conserva sentido en el plano de la responsabilidad por culpa, entonces una condición elemental de su procedencia consiste en que el servicio haya podido comportarse de acuerdo con el estándar exigible. Con todo, la condición económica del hospital está normalmente correlacionada con estándares de servicio diferenciados. En el sistema legal vigente hay tres categorías de establecimien$\operatorname{tos}^{19}$, sin contar con aquellos de la red primaria, destinados a brindar atenciones mucho menos complejas. Es a ese nivel que deberían hallarse las definiciones sobre el equipamiento normal o esperable de cada establecimiento hospitalario.

Por último, la falta de servicio no opera de modo automático cada vez que falta un equipo del inventario del hospital; hay que atender igualmente a las gestiones del servicio en orden a repararlo, reemplazarlo o encontrar un equivalente por otros medios.

\section{El caso de las infecciones intrahospitalarias}

La defensa característica de los servicios de salud respecto de las infecciones intrahospitalarias (entendiendo por tales aquellas contraídas al interior del hospital, sin estar presentes o en incubación antes del ingreso del paciente) consiste en la imposibilidad de erradicarlas. En otros términos, el argumento supone que el recinto hospitalario tiene por rasgo consustancial albergar ciertos factores patógenos que ni con los mayores esfuerzos pueden ser eliminados. En tales circunstancias, cabría deducir, la responsabilidad operaría con prescindencia de las precauciones tomadas para evitar el dańo, esto es, como una responsabilidad

\footnotetext{
19 Siguiendo orientaciones trazadas por el legislador - Ley $N^{\circ} 19.937$ que modifica el D.L. No 2.763, de 1979, con la finalidad de establecer una nueva concepción de la autoridad sanitaria, distintas modalidades de gestión y fortalecer la participación ciudadana (D.O 24 de febrero de 2004) — , un reglamento - Decreto $N^{\circ} 140$ (2005). Reglamento orgánico de los Servicios de Salud. Ministerio de Salud (D.O. 21 de abril de 2005) — establece que todos los hospitales públicos "se clasificarán en establecimientos de alta, mediana o baja complejidad, de acuerdo a su capacidad resolutiva", atendiendo a criterios como su función al interior de la red, los "servicios de apoyo diagnóstico y terapéutico [con que cuentan,] considerando su resolutividad, disponibilidad de recurso humano, equipamiento, horario de atención y procedimientos o exámenes que realiza” y la "especialización de sus recursos humanos" (art. 44). La clasificación respectiva se efectúa caso a caso por el Ministerio de Salud a proposición del respectivo servicio de salud (art. 45).
} 
objetiva o sin culpa ${ }^{20}$ (solución contraria al diseño legal).

Este planteamiento no ha sido acogido por la jurisprudencia. En cambio, ésta ha privado de especificidad al problema de las infecciones nosocomiales, abordándolo desde la perspectiva de los cuidados médicos o de otro orden. El hospital debe prevenir la ocurrencia de estas infracciones; luego, el contagio sufrido por un paciente debe ser detectado y enfrentado a tiempo. De lo contrario, podría configurarse una falta de servicio de vigilancia ${ }^{21}$, de diagnóstico ${ }^{22}$ o de tratamien$\mathrm{to}^{23}$, conforme a las orientaciones generales de la materia.

Esta es una solución institucional y políticamente conservadora. Si resulta plausible el argumento de la inevitabilidad estadística de las infecciones, debiera concluirse que hay algo aleatorio en su materialización en concreto: cualquier persona corre un riesgo importante de infectarse al ingresar a un quirófano. Ahora bien, antes que una excusa, el argumento muestra que hay algo inaceptable en ese riesgo, que se impondría como una especie de gravamen sobre los pacientes y usuarios del servicio público. Siguiendo orientaciones del Derecho comparado, una solución razonable para casos de gravámenes desigualmente repartidos, que guarda conexión con el principio de solidaridad, consiste en una indemnización independiente de la idea de culpa. Con todo, dada la extensión previsible de un régimen de esta especie (que comprenda todos los hospitales públicos y quizá privados) y su impacto en las finanzas públicas, la cuestión debería discutirse en el ámbito legislativo.

\section{A modo de conclusión}

Esta tentativa de categorización de las culpas no médicas de los hospitales públicos muestra la relativa marginalización de los deberes individua-

\footnotetext{
${ }^{20}$ Esta parecía ser la solución a que propendía el fallo de la Corte de Apelaciones de Concepción, de 10 de agosto de 2000, Rol 1.9771999, al declarar que el hospital responde con independencia de sus esfuerzos. Sin embargo, el criterio fue desmentido por la Corte Suprema, 24 de enero de 2002, Rol 3.665-2000.

${ }^{21}$ Corte Suprema, 6 de noviembre de 2017, Rol 62.104-2016.

${ }^{22}$ Corte Suprema, 14 de julio de 2015, Rol 4.156-2015.

23 Corte Suprema, 27 de febrero de 2018, Rol 21.599-2017 (infección a raíz de la instalación fallida de un catéter).
}

les, correlativa al fortalecimiento de los deberes del hospital como institución. A priori, esta configuración de la responsabilidad plantea el desafío de fortalecer la perspectiva de servicio público en la atención de pacientes, de modo que los imperativos éticos infiltren aun en los procedimientos más sencillos, así como en la disposición de los recursos e infraestructuras hospitalarias.

En el plano jurídico, el análisis muestra la extrema ubicuidad del criterio de la falta de servicio, como elemento central del régimen de responsabilidad pecuniaria de los hospitales públicos. En general, el establecimiento de la falta de servicio parece simple, atendida la simplicidad con que la jurisprudencia concibe los deberes exigibles del personal paramédico y administrativo de los hospitales; incluso, tratándose de la responsabilidad derivada de defectos o insuficiencia de los insumos médicos, el relativo automatismo de la reparación sugiere la presencia de una presunción de falta de servicio. Se trata de faltas de servicio comparativamente mucho más fáciles de acreditar que las culpas médicas, en que la lex artis como patrón de referencia suele dejar a la prudencia del especialista algún margen de apreciación sobre el diagnóstico o el tratamiento.

Por cierto, en la medida que la falta de servicio se imputa teóricamente al servicio como un todo, la relativa simplicidad de reconocimiento de la culpa no médica propende, en la estrategia de las víctimas, a la formulación de imputaciones globales, que alcancen tanto al acto médico como a la intervención de otros agentes o de los distintos componentes de la atención hospitalaria ${ }^{24}$.

Conflictos de interés: el autor no tiene conflictos de interés.

\footnotetext{
${ }^{24} \mathrm{La}$ Corte Suprema ha confortado esta estrategia, al emplear una formulación narrativa recurrente que evita identificar específicamente la culpa que origina la responsabilidad, evocando, en cambio, "una serie de hechos, los que analizados en su conjunto, permiten tener por configurada la falta de servicio... [pues el hospital no otorgó al usuario] la atención de salud de manera eficiente y eficaz". La fórmula transcrita parece remontar a un voto disidente en Corte Suprema, 30 de julio de 2012, Rol 355-2010.
} 
Culpas no médicas de los hospitales públicos - José Miguel Valdivia

\section{Referencias}

1. Beauchamp T, Childress J. Principles of Biomedical Ethics. Oxford y Nueva York: Oxford University Press; $4^{\mathrm{a}}$ ed.; 1994.

2. Cárdenas H, Moreno J. Responsabilidad médica. Estándares jurisprudenciales de la falta de servicio. Santiago de Chile: Legal Publishing; 2011.

3. Valdivia JM. La culpa médica en la responsabilidad de los hospitales públicos. Revista Médica de Chile, 2018; 146: 1028-1032.

4. Barros E. Tratado de responsabilidad extracontractual. Santiago: Editorial Jurídica; 2006.

Recibido: 16 de mayo de 2019

Aceptado: 6 de julio de 2019 